\title{
Predicting COVID-19 Dynamics Using SEIR-PADC Model
}

\author{
Ahmad Sedaghat $^{1^{*}}$, Amir Mosavi ${ }^{2,3^{*}}$ \\ ${ }^{1}$ School of Engineering, Australian College of Kuwait, Safat 13015, Kuwait; a.sedaghat@ack.edu.kw; \\ ${ }^{2}$ School of Economics and Business, Norwegian University of Life Sciences, 1430 Ås, Norway \\ ${ }^{3}$ John von Neumann Faculty of Informatics, Obuda University, 1034 Budapest, Hungary; \\ amir.mosavi@kvk.uni-obuda.hu
}

\begin{abstract}
There are a number of derivates of SIR type models developed in mathematics community with 5 to 8 ordinary differential equations to include detailed mechanisms. These models have included exposed, deceased, super-spreader, symptomatic and asymptomatic infected and hospitalized populations; but are mathematically complex and cumbersome. These methods rarely used actual clinical data in details and usually fitted with one or maximum two major clinical data. In this paper, we introduce SEIR-PADC model to include exposed, deceased, super-spreader and critical populations and divide infected population to symptomatic and asymptomatic. SEIR-PADC model is a set of 8 ordinary differential equations with 12 unknown coefficients. Along with, we used an optimization algorithm in MATLAB to find best fit coefficients to 5 set of COVID-19 data in Kuwait. Our focus is to track trends of COVID-19 in coming days. Initial conditions for 8 populations and initial guess values for 12 unknown coefficients are found in a way to best fit COVID-19 data. We used 136 days of COVID-19 data in Kuwait and obtained solutions to cumulative populations rather than daily population. Predictions for 5 different population of COVID-19 in Kuwait using SEIR-PADC model are promising and are discussed here.
\end{abstract}

Keywords: SARS-CoV-2; Coronavirus disease; COVID-19; Dynamics; Kuwait; SIR model; prediction 


\section{Introduction}

In 2012, the Middle Eastern respiratory syndrome coronavirus (MERS-CoV) was reported with largest impact in Saudi Arabia, United Arab Emirates, and South Korea which caused more than $35 \%$ mortality rate among infected people with fever, cough, and shortness of breath [1, $2]$.

This raised concerns in the largest gathering event (Hajj) due to super-spreaders population from the locals infected by MERS-CoV. Alasmawi et al. [3] studied MERS-CoV by an extended SIR model with 5 set of ordinary differential equations for two main subcategories: local population and non-local pilgrims. The 5 set of population include susceptible (S), infected $(\mathrm{I})$, super-spreaders $(\mathrm{P})$, recovered $(\mathrm{R})$, and hospitalized $(\mathrm{H})$. Their study focused on finding mathematically the re-production number $\left(\mathrm{R}_{0}\right)$ and they model coefficients merely found from literature.

Kim et al. [4] studied MERS-CoV which caused the major outbreak in South Korea in 2015. They used a similar extended SIR model as [3] except with 6 set of ordinary differential equations adding asymptomatic infected population (A) with emphasis in determining transmission rates using estimation or literature. Only one set of clinical data on cumulative incidence numbers were used for model validation suggesting that the super spreader population caused initial large reproduction number. They concluded preventive measures such as intensive lockdown and quarantine hospitalized cases had major impact on stopping spread of the MERS-CoV outbreak.

Ndaïrou et al. [5] have recently applied the same extended SIR type model introduced in [3, 4] for studying outbreak of COVID-19 in Wuhan, China with emphasis in stability of their model with 8 set of ordinary differential equations including mortality rates. They fitted their model 
with only two set of clinical data including confirmed daily cases and confirmed daily death cases in Wuhan, China. They estimated model coefficients with values so that a reproduction number below the value of one is obtained. The SIR type models are usually designed for fitting with cumulative clinical data than daily data.

More recently, Xue et al. [6] developed a 7 set of equations SIR type model using a transmission network to fit COVID-19 clinical data in Wuhan (China), Toronto (Canada), and the Italy. They used an optimization algorithm (MCMC) to fit coefficients of 7 set of ODE equations. They only their model with two set of clinical data, confirmed total infected cases and confirmed active infected cases.

Observing the above studied literature, it is not clear why 5 to 8 set of ordinary differential equations are used to only fit with one or maximum two clinical data. From recent COVID-19 data, it can be easily seen that at least 5 set of different clinical data including susceptible, infected, recovery, critical and deceased populations can be used for validation of extended SIR type models. In this paper, we introduce SEIR-PADC which is SIR type model with 8 set of ODE equations. The aim of the paper is to computationally study the 5 set of clinical data on COVID-19 in Kuwait [7] simultaneously to be fitted by SEIR-PADC model using optimization technique in MATLAB (fminsearch tool) and measure clinical significance on development of COVID-19 in Kuwait.

\section{Methodology}

\section{SEIR-PADC Model}

We introduce here SEIR-PADC model consist of susceptible (S), exposed (E), symptomatic infected (I), recovered (R), super spreaders (P), asymptomatic infected (A), deceased (D), and critical (C) populations. The model consist of 8 different cumulative populations and 
transmission from each population starts similar to original SIS and SIR model reported by Kermack andMcKendrick [8]. A flow chart of SEIR-PADC model is shown in Figure 1.

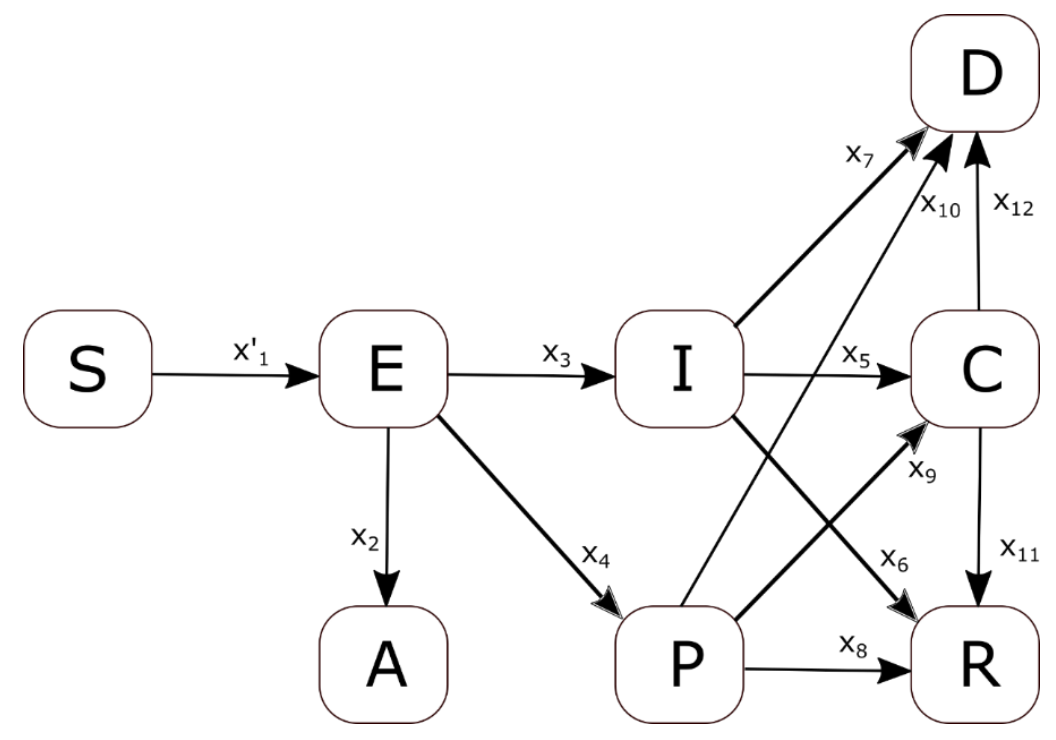

Figure 1: Flow chart of SEIR-PADC dynamic model.

The model have 12 unknown transmission coefficients $\mathrm{x}=\left[\mathrm{x}_{1} \mathrm{x}_{2} \ldots \mathrm{x}_{12}\right]$, which all are positive or negative constants and are obtained by an optimization algorithm to fit clinical data. In Figure 1, $\mathrm{x}^{\prime}{ }_{1}=\mathrm{x}_{1} \mathrm{E} / \mathrm{N}$ is selected as introduced in [8] and flow in and out of each population is simply expressed as follows:

$$
\begin{gathered}
\frac{d S}{d t}=-\frac{x_{1}}{N} S E \\
\frac{d E}{d t}=\frac{x_{1}}{N} S E-x_{2} E-x_{3} E-x_{4} E \\
\frac{d I}{d t}=x_{3} E-x_{5} I-x_{6} I-x_{7} I \\
\frac{d P}{d t}=x_{4} E-x_{8} P-x_{9} P-x_{10} P \\
\frac{d A}{d t}=x_{2} E \\
\frac{d C}{d t}=x_{5} I+x_{9} P-x_{11} C-x_{12} C \\
\frac{d R}{d t}=x_{6} I+x_{8} P+x_{11} C
\end{gathered}
$$




$$
\frac{d D}{d t}=x_{7} I+x_{10} P+x_{12} C
$$

The total population $N$ is constant and is defined by:

$$
N=S+E+I+P+A+C+R+D=\text { constant }
$$

Kuwait has a population around 4,270,571 based on Worldometer 2020 [9]. A set of initial conditions are applied for the 8 populations and 12 coefficients to solve the set of 8 ODE equations (1-8) as follows:

$$
\begin{aligned}
& S_{0}=1200,000 ; E_{0}=5 ; I_{0}=0 ; P_{0}=5 ; A_{0}=0 ; C_{0}=0, R_{0}=0 ; D_{0}=0 \\
& x_{0}=\left[\begin{array}{llllllllllll}
1 & 1 & 1 & 0 & 0 & 1 & 0 & 0 & 2 & 0 & 0 & 1
\end{array}\right]
\end{aligned}
$$

The SEIR-PADC model outlined above are solved using ode45 in MATLAB [10]. The optimization tool (fminsearch) [11] is used to find best fitted ODE coefficients by applying the following convergence criteria for 5-set of available data on goodness of fit (GOF):

$$
\begin{gathered}
\text { GOF }=\operatorname{Minimize}(\operatorname{RMSR}(S)+R M S R(I)+R M S R(R)+R M S R(D) \\
+R M S R(C))
\end{gathered}
$$

In equation (12), the root mean-square ratio (RMSR) of computed population versus available COVID-19 population are calculated and the optimization algorithm (fminsearch) in MATLAB is used to minimize the summation. This is discussed next.

\section{Goodness of fit technique}

\section{The root mean-square ratio}

The root mean-square ratio (RMSR) is defined here using the coefficient of determination which is widely used on goodness of fit when predicted variables are compared with actual 
data particularly for situations that future outcomes are sought. The coefficient of determination $\left(\mathrm{R}^{2}\right)$ evaluates the predicted values $(y)$ against actual data $\left(y_{\text {exp }}\right)$ and is rewritten to provide RMSR as follows $[12,13]$ :

$$
\operatorname{RMSR}=1-\mathrm{R}^{2}=\frac{\sum\left(y-y_{\text {exp }}\right)^{2}}{\sum\left(y_{\text {exp }}-\overline{y_{\text {exp }}}\right)^{2}}
$$

In equation (13), $\overline{y_{\text {exp }}}$ is the average of COVID-19 clinical data value for certain population. Better fitted functions provide $\mathrm{R}^{2}$ value close to unity and RMSR close to zero.

\section{Results of SEIR-PADC model}

Using the optimization algorithm (fminsearch) in MATLAB and initial conditions given in equations (10-11), the following values are found for the model coefficients:

$$
\begin{gathered}
x=\left[\begin{array}{cccccccc}
1.9150 & 1.0159 & 0.8187 & 0.0014 & 0.0008 & 0.0499 & -0.0000 \\
& -0.0003 & 0.4009 & 0.0030 & 0.0032 & 0.0403
\end{array}\right]
\end{gathered}
$$

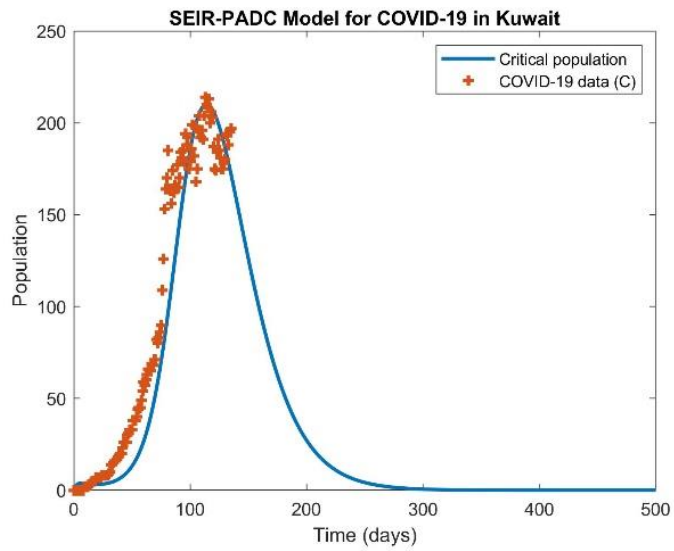

(a) Critical population

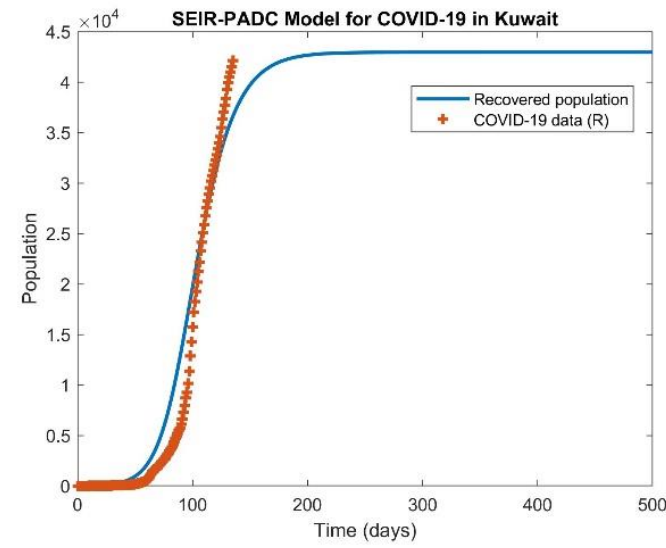

(e) Recovered population 


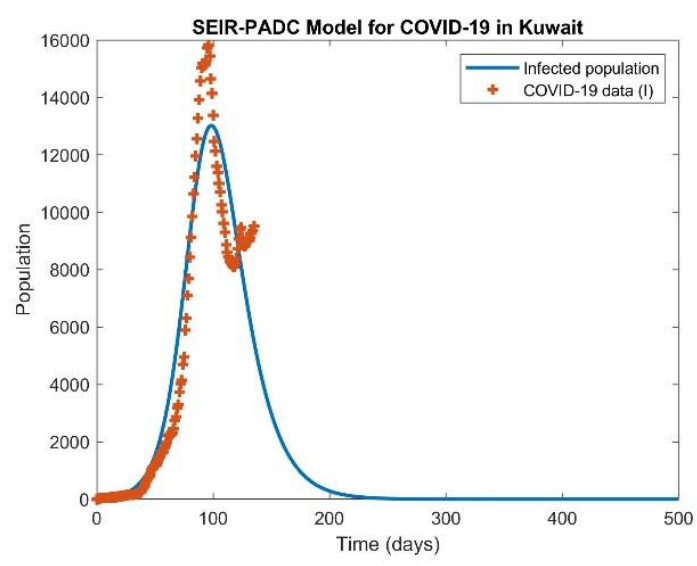

(b) Infected population

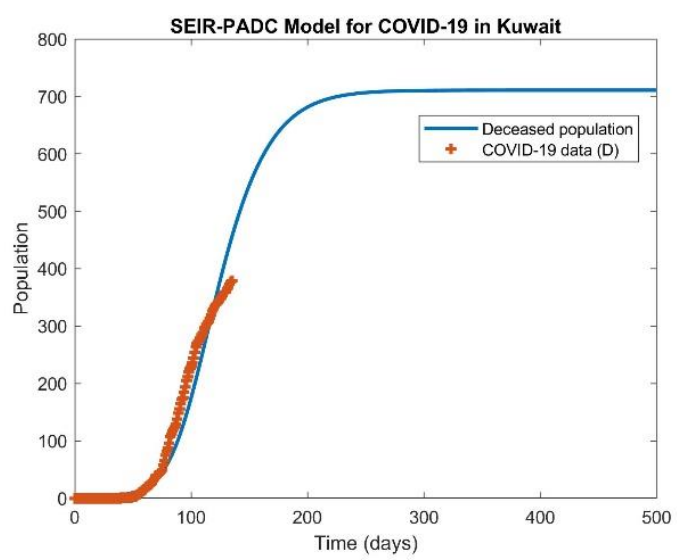

(c) Deceased population

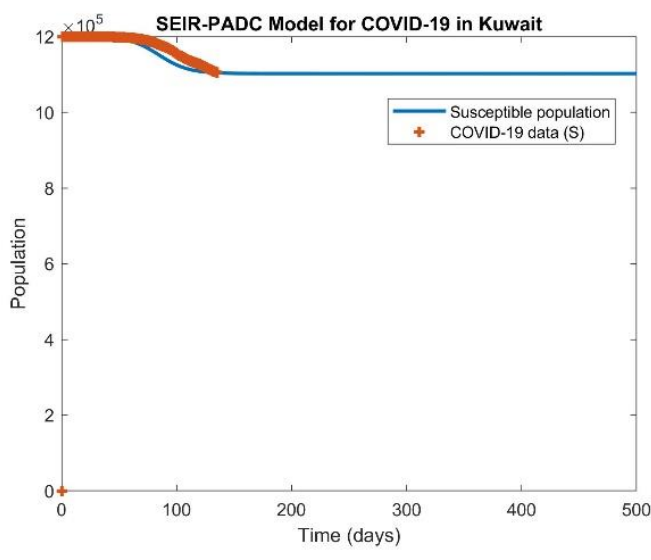

(f) Susceptible population

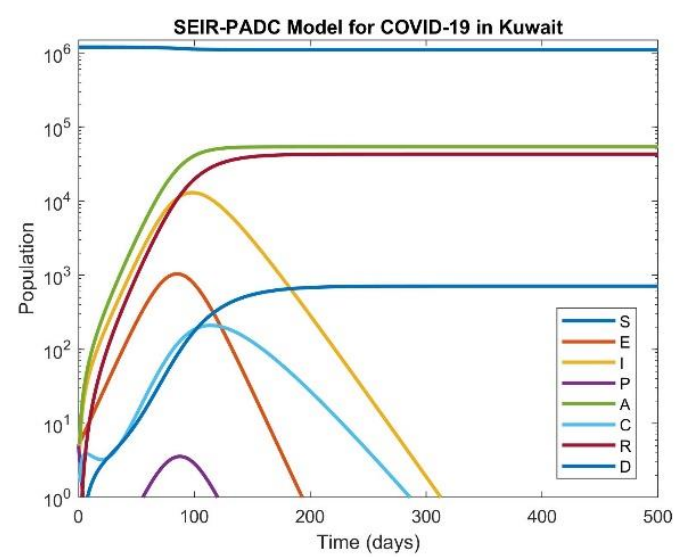

(g) All populations in SEIR-PADC model

Figure 2: Fitting 5 set of COVID-19 data simultaneously with SEIR-PADC model for dynamics of COVID-19 in Kuwait (8 July 2020).

Figure 2 shows that 5 set of COVID-19 data including critical, infected, deceased, recovered, and susceptible populations are simultaneously fitted with SEIR-PADC model. Goodness of fit value (GOF) is obtained 1.5756 for 5 -set of data in equation (12).

Important dates including peak number and day of infectious, peak value and day of people in critical condition, maximum recovered and deceased populations, and end of pandemic in Kuwait can be feasibly obtained from the Figure 2 and computed values from SEIR-PADC model.

\section{Conclusions}


We have developed SEIR-PADC dynamic model for COVID-19 in Kuwait. The aim is to computationally fit simultaneously any available set of COVID-19 data for better prediction of trends of COVID-19 dynamics in Kuwait. From the results of this study, it can be concluded that:

- SEIR-PADC dynamic model was successfully implemented.

- Initial conditions for 12 unknown coefficients in SEIR-PADC model were correctly identified.

- MATLAB optimization with a convergence criterion is successfully applied to find best fitted prediction from SEIR-PADC model to COVID-19 data in Kuwait.

- Important peaks of population sizes and dates are predicted successfully indicting that Kuwait has passed all important peaks of critical hospitalization and peak of infectious populations.

SEIR-PADC model is a simple and robust model to correctly pick up various well-defined population in SIR type models. The model has successfully fitted 5-set of COVD-19 data in Kuwait.

\section{References}

1. Modjarrad K. Treatment strategies for Middle East respiratory syndrome coronavirus. J Virus Erad 2016; 2: 1-4.

2. Centers for Disease Control and Prevention. A Novel Coronavirus Called MERS- CoV [Updated August 22,2016]. Available from: https://www.cdc.gov/coronavirus/

3. Alasmawi H, Aldarmaki N, Tridane A. Modeling of a super-spreading event of the MERScorona virus during the Hajj season using simulation of the existing data. Int J Stat Med Biol Res 2017;1:24-30. 
4. Kim Y, Lee S, Chu C, Choe S, Hong S, Shin Y. The characteristics of middle eastern respiratory syndrome coronavirus transmission dynamics in South Korea. Osong Public Health Res Perspect 2016;7:49-55. doi:10.1016/j.phrp.2016.01.001.

5. Faïçal Ndaïrou, Iván Area, Juan J. Nieto, Delfim F.M. Torres, Mathematical modeling of COVID-19 transmission dynamics with a case study of Wuhan, Chaos, Solitons \& Fractals, Volume 135, 2020, 109846.

6. Ling Xue, Shuanglin Jing, Joel C. Miller, Wei Sun, Huafeng Li, José Guillermo EstradaFranco, James M. Hyman, Huaiping Zhu, A data-driven network model for the emerging COVID-19 epidemics in Wuhan, Toronto and Italy, Mathematical Biosciences, Volume 326, 2020, 108391.

7. COVID-19 updates, State of Kuwait live, https://corona.e.gov.kw/En/, accessed 27 May 2020.

8. Kermack, W. O. and McKendrick, A. G. "A Contribution to the Mathematical Theory of Epidemics.” Proc. Roy. Soc. Lond. A 115, 700-721, 1927.

9. Worldometer, https://www.worldometers.info/world-population/kuwait-population/, accessed 8 July 2020.

10. MathWorks, https://www.mathworks.com/help/matlab/ref/ode45.html, accessed 8 July 2020.

11. MathWorks, https://www.mathworks.com/help/matlab/ref/fminsearch.html, accessed 8 July 2020.

12. Devore, Jay L. (2011). Probability and Statistics for Engineering and the Sciences (8th ed.). Boston, MA: Cengage Learning. pp. 508-510. ISBN 978-0-538-73352-6.

13. Mahmoudi, M.R., Heydari, M.H., Qasem, S.N., Mosavi, A. and Band, S.S., 2020. Principal component analysis to study the relations between the spread rates of COVID-19 in high risks countries. Alexandria Engineering Journal. 